Revista Búsqueda, Vol. 4 Núm. 19:191-207; 2017. ISSN: 0123-9813

DOI: doi.org/10.21892/01239813.371

ORIGINAL

\title{
Factores prenatales y perinatales asociados al coeficiente intelectual en niños y niñas de tres a seis años, del barrio Uribe Uribe, que asisten a hogares infantiles en la ciudad de Sincelejo (Sucre)
}

\section{Prenatal and perinatal factors associated with the iq in children from three to six years of the neighborhood Uribe Uribe ward that assists children's homes in the city of Sincelejo (Sucre)}

\author{
Rohnal José Rada-Luna1 ${ }^{1}$ Katy Estela Arroyo-Alvis², Lisbeth Margarita Hoyos-Cordoba³, \\ Andrés Fernando Ramírez-Giraldo ${ }^{4}$
}

\begin{abstract}
${ }^{1}$ Psicólogo por la Corporación Universitaria del Caribe - CECAR, Colombia. Identificador de autor: http:// orcid.org/ 0000-0001-9141-3473; correo: rohnal.rada@cecar.edu.co

${ }^{2}$ Doctoranda y Magíster en Neuropsicología. Psicóloga. Docente investigadora en Corporación Universitaria del Caribe - CECAR, Colombia. Identificador de autora: http://orcid.org/0000-0003-3171-0702; correo: katy.arroyoa@cecar.edu.co

${ }^{3}$ Magíster en Trastornos Cognoscitivos y del Aprendizaje. Psicóloga. Docente en Corporación Universitaria del Caribe - CECAR, Colombia. Identificador de autora: http://orcid.org/0000-0002-0784-6039; correo: lisbeth.hoyosc@cecar.edu.co

${ }^{4}$ Doctor en Psicología con énfasis en Neurociencias Cognitivas Aplicadas. Psicólogo. Docente investigador en Corporación Universitaria del Caribe - CECAR, Colombia. Identificador de autor: http://orcid.org/00000002-4709-9891; correo: andres.ramirez@cecar.edu.co
\end{abstract}

Recibido: 04-11-2017; Revisado: 23-11-2017; Aceptado: 09-12-2017

\section{Resumen}

La presente investigación tuvo como objetivo determinar la relación entre los factores prenatales y perinatales con el coeficiente intelectual (CI) en niños y niñas del barrio Uribe Uribe de Sincelejo. La metodología de esta investigación fue de un diseño cuantitativo, de tipo observacional y nivel correlacional; constituido por una muestra de 43 niños y niñas en edades de tres a seis años, que asisten a hogares infantiles distribuidos en dos grupos: el grupo uno, con CI menor de 70 y el dos con CI mayor de 70. También se contó con la participación de sus madres. Los instrumentos fueron el Cuestionario Materno de Riesgo Perinatal (CMRP) y la Escala de Inteligencia de Wechsler para Preescolar y Primaria - IV (WPPSI-IV) para evaluar el CI en los niños y niñas. Los resultados del estudio revelaron que no existe diferencia estadística significativa con las variables estudiadas entre los grupos. Se concluyó que no se presenta una asociación en referencia a los factores prenatales y perinatales con el CI en los niños y niñas de la ciudad de Sincelejo.

Palabras clave: coeficiente intelectual, factores prenatales y perinatales, estrato socioeconómico bajo. 


\section{Abstract}

The objective of this investigation was to determine the relationship between prenatal and perinatal factors with the intellectual coefficient (IQ) in children of the Uribe Uribe neighborhood of Sincelejo. The methodology of this investigation was of a quantitative paradigm, of observational type and correlational level; constituted by a sample of 43 boys and girls aged three to six years old, who attend children's homes divided into two groups: the group one with CI less than seventy and the two with an IQ greater than seventy. We also had the participation of their mothers. The instruments were the Maternal Perinatal Risk Questionnaire (CMRP), and the WPPSI-IV, to evaluate IQ in children. The results of the study revealed that there is no significant statistical difference with the variables studied between the groups evaluated. It was concluded that no association is presented in reference to prenatal and perinatal factors with IC in boys and girls in Sincelejo.

Key words: intellectual coefficient, prenatal and perinatal factors, low socioeconomic status.

\section{Introducción}

El desarrollo de la inteligencia ha sido un tema bastante debatido e investigado a lo largo de los últimos años, tanto por su complejidad como por la trascendencia que alcanza como parte fundamental en el desarrollo de las personas (Villatoro, 2016). Tanto es así, que se ha intentado clasificar a los individuos en diversas categorías de inteligencia, dependiendo de la observación del comportamiento de una persona en los diversos aspectos psicológicos, como los rasgos de la personalidad, las habilidades, capacidades mentales, el conocimiento general y actitud, entre otros. Esto acontecía incluso antes de que se inventaran las pruebas que median la inteligencia (Moreno, Yáñez, Prieto, Rodríguez y García, 2017). Hoy en día los diferentes tipos de test de inteligencia se basan en diferentes marcos teóricos y concepciones de la misma; sin embargo, es común que estas herramientas de medida incluyan puntuaciones sobre ámbitos de competencia específicos (lenguaje, inteligencia espacial, etc.) en varios niveles de abstracción, y asimismo, un valor total como dato que integra la inteligencia general del individuo. A este valor se le denomina "coeficiente intelectual" o "cociente intelectual" (CI), que es una medida numérica estadística que determina el nivel de inteligencia de un individuo y se conoce través de la realización de un instrumento estandarizado en relación con el grupo de edad de un sujeto. Se considera que las escalas de Wechsler y StanfordBinet son instrumentos mundialmente reconocidos, diseñados para valorar la inteligencia (Ferrer y Torres, 2014).

Al contrario de lo que se suele pensar comúnmente, el CI no es la inteligencia de una persona, sino un estimador numérico de inteligencia general, por lo que se considera que "la inteligencia y el CI son diferentes puesto que la primera incluye aspectos cualitativos mientras el segundo aspectos cuantitativos" (Villatoro, 2016, p. 14). El CI, propuesto por David Wechsler (1973), es la relación entre la puntuación alcanzada por un individuo en un test de inteligencia y la puntuación que es posible suponer que alcanzará un individuo promedio de su edad natural en el mismo test, cuando ambas anotaciones se expresan 
en la misma anotación $\mathrm{CI}=$ (anotación alcanzada o efectiva) / (anotación media para la edad esperada). El CI propuesto por este autor tiene como característica una constancia en el tiempo, es decir que es perdurable con el paso de los años, siempre y cuando no se presente algún factor externo que haga que cambie, como una lesión cerebral. Esto se debe a que los puntajes arrojados en la escala son relativos y se definen con respecto a la misma inteligencia. Es por esto que se plantea que es necesario que los puntajes de coeficiente intelectual sean independientes de la edad, ya que, el paso de los años no representa un cambio en el CI (Wechsler, 1973).

Por su parte se considera factor de riesgo a todo evento en cuya presencia aumenta la probabilidad de la existencia de una alteración o daño al organismo en cualquiera de sus dimensiones (Harmony, 1996). Sin embargo, esta autora enfatiza en la multiplicidad y simultaneidad de eventos constitutivos de riesgo que pueden confluir en la gestación y nacimiento del ser humano, el cual obliga a la utilización sistemática de métodos estadísticos para establecer relaciones de riesgo y diferenciarla de situaciones causales.

Los Factores de riesgo prenatales, hacen referencia a que, durante el embarazo, pueden presentarse situaciones anómalas que de no controlarse generan afectación en el SNC y la conducta. De acuerdo con Harmony (1996), situaciones tales como la desnutrición, el consumo de alcohol, de tabaco, el uso de medicamentos, la exposición a químicos en el ambiente, a radiaciones y a distintas enfermedades son factores de riesgo importantes.

Los factores de riesgo perinatales, son aquellos presentes en el momento del parto, el cual es otro espacio de tiempo determinante para el futuro de la salud neurológica y psicológica del ser que nace, dadas sus difíciles condiciones naturales. En este periodo llamado perinatal, el cuerpo del infante es sometido a extremo estrés, y se ponen a prueba todas sus capacidades adaptativas, frente a la abrupta interrupción del soporte de vida en oxigenación y nutrición que le brinda el cuerpo de la madre y el estrecho corredor de salida al mundo exterior, paso por el cual, la integridad física de la cabeza fetal es amenazada por la superposición de sus huesos al salir, y la oxigenación general se pone en riesgo por efecto de las contracciones en partos prolongados y otras complicaciones obstétricas (Harmony, 1996). Por otra parte, existen registros de que agentes químicos presentes en el evento del nacimiento que pueden ejercer una influencia en el desarrollo del ser que nace. Por otra parte, Landon, et al., (2004), es claro en referir que la cesárea es un factor de riesgo importante, al igual que las semanas de gestación al momento del parto, como elemento predictor de la talla del bebe.

En términos generales, se puede decir que todo lo que puede causar daño en la mujer durante en el embarazo repercute en el bienestar de esta y del neonato (Papalia, Wendkos y Duskin, 2009), ya que en ese periodo es vulnerable a los factores teratogénicos.

Con el fin de llevar a cabo un adecuado control prenatal y perinatal, los diferentes centros de salud brindan servicios de cuidado, prevención y detección temprana de las alteraciones de embarazos (Ministerio de Salud, 2013); no obstante, las mujeres embarazadas expuestas a deficiencias nutricionales, psicoculturales y socioeconómicas pueden presentar una serie de alteraciones a nivel prenatal y perinatal, como los nacimientos de niños y niñas con alteraciones físicas y cognitivas, con generación de problemas 
en el sistema nervioso, como el retardo madurativo y disminución en el CI (Valenzuela y Trujillo, 1984).

En el departamento de Sucre, el 51,5\% de los habitantes vive en condiciones de pobreza (Departamento Administrativo Nacional de Estadística, 2012). Estos habitantes - especialmente las mujeres embarazadas - están expuestas a complicaciones que se producen durante el embarazo y el parto, o después de ellos. La mayoría de esas complicaciones aparecen durante la gestación y son prevenibles o tratables; otras pueden estar presentes desdeantes del embarazo, pero se agravan con la gestación, especialmente si no se tratan como parte de la asistencia sanitaria a la mujer, lo cual puede presentar consecuencias para el o la bebé (Ministerio de Salud, 2016).

Actualmente, en Sincelejo, la falta de prevención, controles prenatales, seguridad alimentaria y nutricional, aseo e higiene durante los meses de gestación, el consumo de sustancias psicoactivas y alcohol, entre otras, se han determinado como posibles causas de las enfermedades congénitas, malformaciones y anomalías cromosómicas (Secretaria de Planeación de Sincelejo, 2016). Por lo tanto, es urgente $e$ inmediato que desde los entes de salud se generen procesos de sensibilización y prevención a mujeres en edad fértil para que se tomen las medidas preventivas desde antes del embarazo, dados los índices tan altos de esta causa de enfermedades de niños y niñas de cero a cinco años.

El barrio Uribe Uribe es un sector que se encuentra ubicado en la zona sur de la ciudad de Sincelejo (Sucre), cuyos habitantes se encuentran expuestos a problemáticas como los malos olores provenientes de los arroyos cercanos, deficiencias de saneamiento básico (acueducto y alcantarillado) e inundaciones por causa de las lluvias, lo que trae consigo problemas de salud para toda la población. Esto se convierte en un problema debido a que son muchas personas las que se encuentran afectadas por la situación, incluyendo los niños y niñas menores de seis años y las mujeres embarazadas. Por otra parte, en dicho sector se encuentran veinticuatro hogares infantiles, que son guarderías encargadas de atender a una población determinada de niños y niñas hasta los cinco años. Allí se ofrecen desayunos y otras comidas que facilitan el bienestar de los niños y niñas mientras los cuidadores se encuentran ausentes debido al trabajo u otras responsabilidades. Esto permite que niños y niñas lleven a cabo en dichas instalaciones actividades acordes a su edad para un buen desarrollo cognitivo.

Existen diversos factores de riesgo prenatales y perinatales que pueden estar asociados al desarrollo del niño y de su inteligencia, como el uso de drogas o alcohol, (Thompson, Levitt y Stanwood, 2009; Jacobson y Jacobson, 2002), la exposición a sustancias tóxicas (Evans y Kantrowitz, 2002), factores ambientales, físicos o sociales (Glover, 2011) y una dieta incorrecta (Rijlaarsdam, et al., 2017).

En lo que respecta al consumo de sustancias durante el embarazo; como es el caso de la exposición prenatal a la cocaína, se ha encontrado que ésta puede traer efectos negativos en facultades cognitivas como la atención y en las funciones ejecutivas (Dow-Edwards, Mayers, Spear y Hurd, 1999). En esa línea, la exposición a las anfetaminas y metanfetaminas provocan un aumento de estrés, repercutiendo directamente sobre el nivel de la atención sostenida y la memoria (Derauf, et al., 2007). La nicotina por su parte, se ha encontrado que afecta principalmente de forma general al encéfalo, asociándolo en ocasiones a patologías del neurodesarrollo 
con el trastorno por déficit de atención e hiperactividad (TDAH) y dificultades de aprendizaje (Lambe, et al., 2006; Langley, Rice, Van den Bree y Thapar, 2005).

En lo que respecta al consumo de alcohol; se ha encontrado que éste, al atravesar la placenta durante el periodo gestacional por compartir fluido sanguíneo, puede traer consecuencias negativas en los recién nacidos como el llamado síndrome de alcoholismo fetal (SAF), que repercute directamente en facultades cognitivas como el lenguaje, la memoria, la atención y el aprendizaje (Feng, Yan y Yan, 2005; Huang, Di Cristo y Ango, 2007; Miller, 2003; Mattson, Crocker y Nguyen, 2011); además de ser un factor relevante en el desarrollo del CI (Zapata, Álvarez, Aguire y Cadavid, 2012).

En base a ese último punto relacionado con los factores prenatales y perinatales asociados al CI, últimamente se ha hecho evidente discrepancias en relación a la influencia entre ambas (Vishnevetsky, et al., 2015; Shen, et al., 1998; Lanphear et al., 2005; Vaurio, et al., 2011; Mahoney, Taylor \& Kanarek, 2005). Por otro lado, otros autores pese a la literatura que relaciona a ambas variables; sus investigaciones han concluido a la poca relacion que guardan los factores prenatales y perinatales en el CI. (Gaspar, et al., 2015; Wasserman, et al., 2000; Schnaas, et al., 2000; Singer, et al., 2005; Frank, et al., 2004).

Retomando lo anterior expuesto, se torna un punto importante sobre el estudio de la incidencia de los factores prenatales y perinatales en el $\mathrm{CI}$; lo que conlleva al interrogante de si es posible que alguno (os) factores prenatales y perinatales puedan estar asociados al CI de los niños del barrio Uribe Uribe- Sincelejo.

\section{Metodología}

Esta investigación se enmarcó en un paradigma positivista, con un método cuantitativo, de tipo observacional y de nivel correlacional. La muestra se realizó de manera intencional y estuvo compuesta por dos grupos: el primer grupo, compuesto por veinte niños y niñas que obtuvieron en la prueba de CI un puntaje menor de 70 y sus respectivas madres. El segundo grupo, compuesto por veintitrés niños y niñas que obtuvieron en la prueba de CI un puntaje mayor a 70 y sus respectivas madres. En ambos grupos se aplicó a las madres el CMRP para describir los aspectos prenatales y perinatales. Estos niños y niñas tenían edades entre los tres y los seis años; en su totalidad eran asistentes a hogares infantiles en el barrio Uribe Uribe en la ciudad de Sincelejo (Sucre).

Para recoger la información acerca del CI en los niños y niñas, se utilizó la WPPSIIV, el cual es un instrumento clínico que se ha diseñado para evaluar las aptitudes intelectuales en niños y niñas con edades comprendidas entre los dos años y seis meses, y los tres años y once meses (2:6 - 3:11). También en niños y niñas con edades comprendidas entre los cuatro años y los siete años y siete meses (4:0 - 7:7). A través de este instrumento se evaluaron aspectos específicos a través de las escalas primarias como el índice de comprensión verbal (ICV), índice visoespacial (IVE), índice de memoria de trabajo (IMT), índice de razonamiento fluido (IRF), índice de velocidad de procesamiento (IVP) y el coeficiente intelectual total (CIT). También, para recoger la información prenatal y perinatal se les aplicó a las madres el CMRP, que es una prueba estructurada que tiene por objetivo recolectar información acerca del periodo de embarazo, en las etapas que comprenden el periodo pregestacional, el periodo neonatal, el periodo intraparto y 
perigestacional, y el periodo demográfico y psicosocial.

La investigación se llevó a cabo en tres fases: primero, se inició con la visita a los hogares infantiles ubicados en el barrio Uribe Uribe, donde se convocó a una reunión a la que asistieron los padres y madres de los niños y niñas. Luego, se explicó el proyecto de investigación, la finalidad, los objetivos y los alcances que presenta para conocer el número de participantes que harían parte de la investigación. Por último, se llevó a cabo la firma del consentimiento informado y, posteriormente, la aplicación de los instrumentos a los niños, niñas y sus respectivas madres. Después de recolectar los datos a partir de los cuestionarios, se creó una base de datos que posteriormente fue analizada en el software de análisis estadístico SPSS (versión 18), para determinar los factores prenatales y perinatales con el CI en niños y niñas. Este análisis se hizo a través de medidas de tendencia central, porcentajes y frecuencia para variables cualitativas en ambos grupos.
Posteriormente se utilizó el chi cuadrado para la comparación de ambos grupos y para poder observar la existencia de diferencias significativas entre ambos.

\section{Resultados}

En las variables preconcepcionales (Tabla 1) no se encontraron diferencias estadísticas significativas entre los dos grupos, aunque los valores que presentaron mayor acercamiento fueron los de la variable peso de la madre antes del embarazo $(p<0,198)$ y los de la variable enfermedades previas al embarazo $(p<0,227)$. En las variables prenatales (Tabla 2) no se encontraron diferencias estadísticas significativas entre los dos grupos. En las variables intraparto (Tabla 3), no se encontraron diferencias estadísticas significativas entre los dos grupos, aunque los valores que presentaron mayor acercamiento fueron la variable anestesia durante el parto $(p<0,067)$ y en las variables posparto (Tabla 4). No se encontraron diferencias estadísticas significativas entre los dos grupos.

Tabla 1. Comparación entre grupo CI menor a 70 y CI mayor a 70 en las variables Preconcepcionales

\begin{tabular}{|c|c|c|c|c|c|}
\hline & \multirow{2}{*}{ Variables } & \multicolumn{2}{|c|}{ Grupo } & \multirow{2}{*}{$\mathbf{C h i}^{2}$} & \multirow{2}{*}{ Sig. } \\
\hline & & $\begin{array}{l}\text { CI menor a } \\
70 \mathrm{~N}(\%)\end{array}$ & $\begin{array}{l}\text { CI mayor a } \\
70 \mathrm{~N}(\%)\end{array}$ & & \\
\hline \multirow{7}{*}{ Peso antes del embarazo } & Menos de $40 \mathrm{Kg}$ & $0(0)$ & $1(100)$ & \multirow{7}{*}{8,584} & \multirow{7}{*}{0,198} \\
\hline & Entre 40 y $50 \mathrm{Kg}$ & $4(44,4)$ & $5(55,6)$ & & \\
\hline & Entre 51 y $60 \mathrm{Kg}$ & $7(41,2)$ & $10(58,8)$ & & \\
\hline & Entre 61 y $70 \mathrm{Kg}$ & $4(44,4)$ & $5(55,6)$ & & \\
\hline & Entre 71 y $80 \mathrm{Kg}$ & $0(0)$ & $1(100)$ & & \\
\hline & Más de $80 \mathrm{Kg}$ & $0(0)$ & $1(100)$ & & \\
\hline & No Recuerda & $5(100)$ & $0(0)$ & & \\
\hline \multirow{4}{*}{$\begin{array}{l}\text { Estatura antes del } \\
\text { embarazo }\end{array}$} & Menos de $150 \mathrm{~cm}$ & $1(100)$ & $0(0)$ & \multirow{4}{*}{1,295} & \multirow{4}{*}{0,730} \\
\hline & Entre 151 y $160 \mathrm{~cm}$ & $10(47,6)$ & $11(52,4)$ & & \\
\hline & Entre 161 y $170 \mathrm{~cm}$ & $7(43,8)$ & $9(56,3)$ & & \\
\hline & No Recuerda & $2(40)$ & $3(60)$ & & \\
\hline
\end{tabular}


Rada-Luna et al. Factores prenatales y perinatales asociados al coeficiente intelectual en niños (a) de tres a seis años

\begin{tabular}{|c|c|c|c|c|c|}
\hline \multirow{4}{*}{$\begin{array}{l}\text { Número de embarazos } \\
\text { anteriores }\end{array}$} & Ninguno & $8(42,1)$ & $11(57,9)$ & \multirow{4}{*}{1,669} & \multirow{4}{*}{0,644} \\
\hline & Uno & $6(42,9)$ & $8(57,1)$ & & \\
\hline & Dos & $5(55,6)$ & $4(44,4)$ & & \\
\hline & Tres & $1(100)$ & $0(0)$ & & \\
\hline \multirow{2}{*}{$\begin{array}{l}\text { Abortos voluntarios } \\
\text { anteriores }\end{array}$} & Ninguno & $19(45,2)$ & $23(54,8)$ & \multirow[b]{2}{*}{1,177} & \multirow[b]{2}{*}{0,278} \\
\hline & Uno & $1(100)$ & $0(0)$ & & \\
\hline Hijos con alteraciones & No & $19(51,4)$ & $18(48,6)$ & 5,846 & 0,441 \\
\hline \multirow{7}{*}{$\begin{array}{l}\text { Problemas en embarazos } \\
\text { anteriores }\end{array}$} & Bajo peso del bebe (menos de 2,500 grs) & $1(33,3)$ & $2(66,7)$ & \multirow{7}{*}{5,484} & \multirow{7}{*}{0,483} \\
\hline & Mortalidad del feto & $1(100)$ & $0(0)$ & & \\
\hline & Bebé prematuro (menos de 37 semanas) & $1(100)$ & $0(0)$ & & \\
\hline & Sobrepeso del bebé (más de 4000 grs) & $0(0)$ & $1(100)$ & & \\
\hline & Cesáreas por posición fetal & $1(33,3)$ & $2(66,7)$ & & \\
\hline & Bajo peso del bebé y bebé prematuro & $0(0)$ & $2(100)$ & & \\
\hline & Ninguna de ellas & $16(50)$ & $16(50)$ & & \\
\hline \multirow{4}{*}{$\begin{array}{l}\text { Cirugías antes / durante } \\
\text { el embarazo }\end{array}$} & Anterior a este embarazo & $2(66,7)$ & $1(33,3)$ & \multirow{4}{*}{2,558} & \multirow{4}{*}{0,465} \\
\hline & Durante este embarazo & $1(100)$ & $0(0)$ & & \\
\hline & Episiotomía anterior a este embarazo & $0(0)$ & $1(100)$ & & \\
\hline & Ninguna & $17(44,7)$ & $21(55,3)$ & & \\
\hline \multirow[t]{2}{*}{ Uso de anticonceptivos } & Anticonceptivos hormonales & $6(54,5)$ & $5(45,5)$ & \multirow[t]{2}{*}{0,383} & \multirow[t]{2}{*}{0,536} \\
\hline & Ninguno & $14(43,8)$ & $18(56,3)$ & & \\
\hline \multirow{5}{*}{$\begin{array}{l}\text { Edad al momento de la } \\
\text { concepción }\end{array}$} & Entre 15 y 20 años & $10(52,6)$ & $9(47,4)$ & \multirow{5}{*}{2,522} & \multirow{5}{*}{0,641} \\
\hline & Entre 21 y 26 años & $8(50)$ & $8(50)$ & & \\
\hline & Entre 27 y 32 años & $2(33,3)$ & $4(66,7)$ & & \\
\hline & Entre 33 y 38 años & $0(0)$ & $1(100)$ & & \\
\hline & Entre 39 y 44 años & $0(0)$ & $1(100)$ & & \\
\hline \multirow{6}{*}{$\begin{array}{l}\text { Enfermedades previas al } \\
\text { embarazo }\end{array}$} & Ninguna & $10(37,0)$ & $17(63,0)$ & \multirow{6}{*}{6,912} & \multirow{6}{*}{0,227} \\
\hline & Anemias frecuentes & $1(50)$ & $1(50)$ & & \\
\hline & Infecciones urinarias & $8(72,7)$ & $3(27,3)$ & & \\
\hline & Enfermedad de la tiroides & $0(0)$ & $1(100)$ & & \\
\hline & Anemias e infecciones urinarias & $0(0)$ & $1(100)$ & & \\
\hline & Infecciones urinarias e hipertensión & $1(100)$ & $0(0)$ & & \\
\hline
\end{tabular}

Fuente: Elaboración propia 
Revista Búsqueda - Julio / Diciembre de 2017

Tabla 2. Comparación de grupo CI menor a 70 y CI mayor a 70 en las variables Prenatales

\begin{tabular}{|c|c|c|c|c|c|}
\hline & \multirow{2}{*}{ Variables } & \multicolumn{2}{|c|}{ Grupo } & \multirow{2}{*}{ Chi $^{2}$} & \multirow{2}{*}{ Sig. } \\
\hline & & $\begin{array}{l}\text { CI menor a } \\
70 \mathrm{~N}(\%)\end{array}$ & $\begin{array}{l}\text { CI mayor a } \\
70 \mathrm{~N}(\%)\end{array}$ & & \\
\hline \multirow{8}{*}{$\begin{array}{l}\text { Peso ganado durante ese } \\
\text { embarazo }\end{array}$} & Menos de $4 \mathrm{Kg}$ & $6(75)$ & $2(25)$ & \multirow{8}{*}{10,882} & \multirow{8}{*}{0,144} \\
\hline & Entre 5 y $7 \mathrm{Kg}$ & $3(33,3)$ & $6(66,7)$ & & \\
\hline & Entre 8 y $11 \mathrm{Kg}$ & $1(14,3)$ & $6(85,7)$ & & \\
\hline & Entre 12 y $15 \mathrm{Kg}$ & $4(80)$ & $1(20)$ & & \\
\hline & Entre 16 y $20 \mathrm{Kg}$ & $0(0)$ & $2(100)$ & & \\
\hline & Más de $20 \mathrm{Kg}$ & $1(33,3)$ & $2(66,7)$ & & \\
\hline & No recuerda & $3(50)$ & $3(50)$ & & \\
\hline & Perdió peso & $2(66,7)$ & $1(33,3)$ & & \\
\hline \multirow{4}{*}{$\begin{array}{l}\text { Primera consulta médica } \\
\text { durante ese embarazo }\end{array}$} & Primer mes de embarazo & $6(46,2)$ & $7(53,8)$ & \multirow{4}{*}{3,521} & \multirow{4}{*}{0,172} \\
\hline & Entre el segundo y tercer mes & $8(36,4)$ & $14(63,6)$ & & \\
\hline & Entre el cuarto y el sexto mes & $6(75)$ & $2(25)$ & & \\
\hline & Total & $20(46,5)$ & $23(53,5)$ & & \\
\hline \multirow{3}{*}{$\begin{array}{l}\text { Sangrado vaginal durante } \\
\text { ese embarazo }\end{array}$} & Nunca & $18(47,4)$ & $20(52,6)$ & \multirow{3}{*}{1,905} & \multirow{3}{*}{0,386} \\
\hline & Algunas veces al principio del embarazo & $1(25)$ & $3(75)$ & & \\
\hline & Algunas veces a lo largo del embarazo & $1(100)$ & $0(0)$ & & \\
\hline \multirow{4}{*}{$\begin{array}{l}\text { Ingesta de medicación } \\
\text { durante el embarazo }\end{array}$} & Suplementos vitamínicos & $2(25)$ & $6(75)$ & \multirow{4}{*}{4,814} & \multirow{4}{*}{0,186} \\
\hline & Acetaminofén durante el embarazo & $2(100)$ & $0(0)$ & & \\
\hline & Varias de las opciones anteriores & $16(50)$ & $16(50)$ & & \\
\hline & Ningún medicamento & $0(0)$ & $1(100)$ & & \\
\hline \multirow{4}{*}{$\begin{array}{l}\text { Consumo de cigarrillo o } \\
\text { marihuana }\end{array}$} & Ninguna de las dos & $19(46,3)$ & $22(53,7)$ & \multirow{4}{*}{2,020} & \multirow{4}{*}{0,364} \\
\hline & Cigarrillo & $0(0)$ & $1(100)$ & & \\
\hline & Marihuana & $1(100)$ & $0(0)$ & & \\
\hline & Total & $20(46,5)$ & $23(53,5)$ & & \\
\hline \multirow[t]{2}{*}{ Consumo de alcohol } & En algunas ocasiones & $7(38,9)$ & $11(61,1)$ & \multirow[t]{2}{*}{0,723} & \multirow[t]{2}{*}{0,395} \\
\hline & Ninguno & $13(52)$ & $12(48)$ & & \\
\hline \multirow{13}{*}{$\begin{array}{l}\text { Enfermedades presentes } \\
\text { durante el embarazo }\end{array}$} & Ninguna & $4(36,4)$ & $7(63,6)$ & \multirow{13}{*}{10,326} & \multirow{13}{*}{0,587} \\
\hline & Enfermedad sanguínea & $1(33,3)$ & $2(66,7)$ & & \\
\hline & Infecciones urinarias & $5(50)$ & $5(50)$ & & \\
\hline & Asma & $1(100)$ & $0(0)$ & & \\
\hline & Hipertensión & $0(0)$ & $2(100)$ & & \\
\hline & Problemas con el líquido amniótico & $0(0)$ & $1(100)$ & & \\
\hline & Fiebre frecuente & $0(0)$ & $1(100)$ & & \\
\hline & Debilidad y desmayos & $2(66,7)$ & $1(33,3)$ & & \\
\hline & Infección vaginal antes del parto & $2(50)$ & $2(50)$ & & \\
\hline & $\begin{array}{l}\text { Infección urinaria e infección vaginal } \\
\text { antes del parto }\end{array}$ & $3(75)$ & $1(25)$ & & \\
\hline & Infecciones urinarias y desnutrición & $1(100)$ & $0(0)$ & & \\
\hline & $\begin{array}{l}\text { Problema con el líquido amniótico y } \\
\text { Infección vaginal antes del parto }\end{array}$ & $0(0)$ & $1(100)$ & & \\
\hline & $\begin{array}{l}\text { Enfermedad sanguínea y problema con } \\
\text { el líquido amniótico }\end{array}$ & $1(100)$ & $0(0)$ & & \\
\hline
\end{tabular}


Rada-Luna et al. Factores prenatales y perinatales asociados al coeficiente intelectual en niños (a) de tres a seis años

Tabla 3. Comparación de grupo CI menor a 70 y CI mayor a 70 en las variables Intraparto

\begin{tabular}{|c|c|c|c|c|c|}
\hline \multirow{2}{*}{\multicolumn{2}{|c|}{ Variables }} & \multicolumn{2}{|c|}{ Grupo } & \multirow{7}{*}{$\begin{array}{c}\mathbf{C h i}^{\mathbf{2}} \\
8,780\end{array}$} & \multirow{7}{*}{$\begin{array}{l}\text { Sig. } \\
0,067\end{array}$} \\
\hline & & \multirow{2}{*}{$\begin{array}{c}\text { CI menor a } \\
70 \mathrm{~N}(\%) \\
4(100)\end{array}$} & \multirow{2}{*}{$\begin{array}{l}\text { CI mayor a } \\
70 \mathrm{~N}(\%) \\
0(0)\end{array}$} & & \\
\hline \multirow{5}{*}{$\begin{array}{l}\text { Anestesia durante } \\
\text { el parto }\end{array}$} & Ninguna & & & & \\
\hline & Anestesia epidural & $11(35,5)$ & $20(64,5)$ & & \\
\hline & Anestesia general & $3(75)$ & $1(25)$ & & \\
\hline & Desconozco si se utilizó algún tipo de anestesia & $2(66,7)$ & $1(33,3)$ & & \\
\hline & Anestesia epidural y refuerzo posterior al parto & $0(0)$ & $1(100)$ & & \\
\hline \multirow{3}{*}{$\begin{array}{l}\text { Al momento de } \\
\text { romper fuente } \\
\text { ocurrió }\end{array}$} & Fue un parto por cesárea & $15(41,7)$ & $21(58,3)$ & \multirow{3}{*}{2,603} & \multirow{3}{*}{0,272} \\
\hline & Me dieron medicación para calmar dolores & $1(50)$ & $1(50)$ & & \\
\hline & Comenzaron las contracciones y al poco tiempo el parto & $4(80)$ & $1(20)$ & & \\
\hline \multirow{4}{*}{$\begin{array}{l}\text { Posición fetal del } \\
\text { niño al momento } \\
\text { del parto }\end{array}$} & De lado, atravesado & $1(100)$ & $0(0)$ & \multirow{4}{*}{1,583} & \multirow{4}{*}{0,663} \\
\hline & De cabeza & $16(47,1)$ & $18(52,9)$ & & \\
\hline & De nalga o de pie & $1(50)$ & $1(50)$ & & \\
\hline & No estoy segura & $2(33,3)$ & $4(66,7)$ & & \\
\hline \multirow{4}{*}{ Duración del parto } & Fue un parto por cesárea & $15(41,7)$ & $21(58,3)$ & \multirow{4}{*}{3,139} & \multirow{4}{*}{0,371} \\
\hline & Entre 1 y 2 horas & $2(100)$ & $0(0)$ & & \\
\hline & Entre 3 y 5 horas & $2(66,7)$ & $1(33,3)$ & & \\
\hline & No recuerda/no sabe & $1(50)$ & $1(50)$ & & \\
\hline \multirow{4}{*}{$\begin{array}{l}\text { Inducción o parto } \\
\text { provocado }\end{array}$} & Antes de la 37 semanas & $1(33,3)$ & $2(66,7)$ & \multirow{4}{*}{1,900} & \multirow{4}{*}{0,593} \\
\hline & Entre la 37 y 41 semanas & $13(54,2)$ & $11(45,8)$ & & \\
\hline & Después de las 41 semanas & $0(0)$ & $1(100)$ & & \\
\hline & No se indujo el parto & $6(40)$ & $9(60)$ & & \\
\hline \multirow{3}{*}{ Fórceps o ventosa } & Fórceps & $1(50)$ & $1(50)$ & \multirow{3}{*}{1,177} & \multirow{3}{*}{0,555} \\
\hline & Ninguno & $4(66,7)$ & $2(33,3)$ & & \\
\hline & Parto por cesárea & $15(42,9)$ & $20(57,1)$ & & \\
\hline
\end{tabular}

Fuente: Elaboración propia

Tabla 4. Comparación de grupo CI menor a 70 y CI mayor a 70 en las variables postparto

\begin{tabular}{|c|c|c|c|c|c|}
\hline \multirow{2}{*}{\multicolumn{2}{|c|}{ Variables }} & \multicolumn{2}{|c|}{ Grupo } & \multirow{2}{*}{ Chi $^{2}$} & \multirow{2}{*}{ Sig. } \\
\hline & & $\begin{array}{l}\text { CI menor a } \\
70 \mathrm{~N}(\%)\end{array}$ & $\begin{array}{l}\text { CI mayor a } \\
70 \mathrm{~N}(\%)\end{array}$ & & \\
\hline \multirow{4}{*}{$\begin{array}{l}\text { Dificultades al } \\
\text { momento del parto }\end{array}$} & Circular del cordón & $1(33,3)$ & $2(66,7)$ & \multirow{4}{*}{0,718} & \multirow{4}{*}{0,869} \\
\hline & Hipóxia & $2(66,7)$ & $1(33,3)$ & & \\
\hline & Ninguna dificultad & $16(45,7)$ & $19(54,3)$ & & \\
\hline & No recuerda & $1(50)$ & $1(50)$ & & \\
\hline \multirow{3}{*}{ Peso del niño al nacer } & Entre 1500 y 2500 grs & $4(80)$ & $1(20)$ & \multirow{3}{*}{2,914} & \multirow{3}{*}{0,233} \\
\hline & Entre 2500 y 3500 grs & $11(45,8)$ & $13(54,2)$ & & \\
\hline & Entre 3500 y 4000 grs & $5(35,7)$ & $9(64,3)$ & & \\
\hline \multirow{3}{*}{$\begin{array}{l}\text { Tiempo de duración del } \\
\text { embarazo }\end{array}$} & Entre 28 y 36 semanas & $1(33,3)$ & $2(66,7)$ & \multirow{3}{*}{1,155} & \multirow{3}{*}{0,561} \\
\hline & Entre 37 y 41 semanas & $19(48,7)$ & $20(51,3)$ & & \\
\hline & Más de 41 semanas & $0(0)$ & $1(100)$ & & \\
\hline \multirow{4}{*}{$\begin{array}{l}\text { Coloración fetal } \\
\text { (morado o rojo) }\end{array}$} & Ninguna coloración & $18(51,4)$ & $17(48,6)$ & \multirow{4}{*}{2,163} & \multirow{4}{*}{0,539} \\
\hline & Por algunas partes del cuerpo & $1(33,3)$ & $2(66,7)$ & & \\
\hline & Por todo el cuerpo & $1(25)$ & $3(75)$ & & \\
\hline & No estoy segura & $0(0)$ & $1(100)$ & & \\
\hline \multirow{3}{*}{$\begin{array}{l}\text { Cuidado especial con el } \\
\text { niño al nacer }\end{array}$} & Incubadora & $1(33,3)$ & $2(66,7)$ & \multirow{3}{*}{0,584} & \multirow{3}{*}{0,747} \\
\hline & Cuidados de urgencia al poco tiempo de nacer & $3(60)$ & $2(40)$ & & \\
\hline & Ninguna & $16(45,7)$ & $19(54,3)$ & & \\
\hline
\end{tabular}




\section{Discusión}

No se encontró asociación entre los factores prenatales y perinatales con el coeficiente intelectual en los niños y niñas de tres a seis años del barrio Uribe Uribe, que asisten a hogares infantiles en la ciudad de Sincelejo (Sucre). Estos resultados coinciden con los encontrados por Cadavid, Zapata, Aguirre y Álvarez, (2011), en los que mencionan que los factores prenatales y perinatales no condicionan directamente los puntajes de coeficiente intelectual, aunque se encuentren muy vinculados con variables que resultan correlacionadas, como el estrato socioeconómico, el nacimiento y el nivel educativo de madres y padres. Por otra parte, los resultados presentados en esta investigación irían en contra con respecto a los resultados obtenidos por Martin y Dombrowski (2008), en los que mencionan que los acontecimientos presentados a nivel prenatal y perinatal influyen en las pruebas de inteligencia afecta en los niños y niñas.

Ahora bien, con respecto a las variables preconcepcionales, se puede apreciar que ninguna de las madres, antes del embarazo, presentó un peso mayor de 71 $\mathrm{Kg}$. Además, se observó que la estatura presentada en las madres antes y durante el embarazo en esta investigación fueron de nivel promedio, ya que se sabe que los promedios de talla y peso de los adultos difieren considerablemente de una población a otra. Esto coincide con los resultados presentados por Goya, Flores, Astudillo, Viso y Cabrero (2008) quienes afirmaron que la talla depende en gran parte de factores genéticos, y no solo de la acción del medio, aparte de su importancia obstétrica y de la importancia general como indicador posible del estado nutricional. Se encontró además una relación con lo planteado por Bolzán y Guimarey (2001), ya que estos parten de la idea de que la estatura de la madre tiene un interés directo para el cálculo de las necesidades alimentarias durante la gestación. Sin embargo, los actuales conocimientos existentes sobre las variaciones de la talla materna durante el embarazo, entre una población y otra, son todavía insuficientes como para considerar la estatura como factor de riesgo a nivel prenatal (Minjarez, et al., 2014). Asimismo, se puede afirmar que el grupo de CI mayor a 70 tuvo mayores problemas de embarazos anteriores, especialmente problemas como el bajo peso del bebé al nacer y que haya sido prematuro.

Por otra parte, se encontró que el método de planificación familiar utilizado por las madres antes del embarazo, para ambos grupos, fue el de los anticonceptivos hormonales. Esto concuerda con el estudio realizado por Arrate, Linares, Molina, Sánchez y Arias (2013), quienes señalan que las mujeres que se encuentran en la etapa de adolescencia son las que mayormente utilizan este tipo de método anticonceptivo. Además, estos autores afirman que antes de utilizar cualquier método anticonceptivo, se debe tener conocimiento de las indicaciones, contraindicaciones y posibles efectos secundarios, con el fin de evitar problemas de salud y complicaciones.

Con respecto a las variables prenatales, en el peso ganado durante el embarazo, se encontró que el mayor índice se presenta entre los $5 \mathrm{Kg}$. y $7 \mathrm{Kg}$. No obstante, el estudio realizado por Minjarez, et al. (2014) encontró que el peso ganado asociado con el embarazo oscila entre 0,5 $\mathrm{Kg}$. y $3,8 \mathrm{~kg}$. El Institute of Medicine (IOM) en el año 1990 registró que el intervalo recomendado de ganancia de peso gestacional total de una mujer durante el embarazo es de $11,5 \mathrm{Kg}$. a $16 \mathrm{Kg}$., ya que la ganancia de peso durante el embarazo incluye los procesos biológicos diseñados para fomentar el crecimiento fetal (San Román, 2013). Sin embargo, no siempre 
es preferible una mayor ganancia de peso (Herring y Oken, 2010). En estudios recientes, se han destacado algunos de los desenlaces negativos de salud asociados a mayores ganancias durante el embarazo (Viswanathan, et al. 2008), entre los que destacan complicaciones del parto (Catalano, 2007), sobrepeso infantil (Olson, Strawderman y Dennison, 2009; Oken, Taveras, Kleinman, Rich-Edwards y Gillman, 2007) y mayor retención del peso postparto, lo que predispone a un riesgo posterior de obesidad en la madre (Olson, 2008; Gunderson y Abrams, 2000).

En cuanto a la variable de primera consulta médica durante ese embarazo, se evidenció que la mayoría de las gestantes la realizaron en el segundo y tercer mes de embarazo en ambos grupos. Esto coincide con los resultados analizados por Sánchez, Pérez, Pérez y Vásquez (2005), quienes encontraron que las mujeres realizaron más de cinco consultas en el primer trimestre del embarazo. La importancia de un control prenatal adecuado radica en que desde el primer mes de embarazo y con consultas médicas una vez por mes hasta el parto, se pueden identificar complicaciones del embarazo como infecciones cervicovaginales, diabetes gestacional, preeclampsia, infecciones sistémicas (VIH y otras), y permiten establecer medidas preventivas oportunas de acuerdo con la situación de la embarazada en el momento oportuno, lo que significa un costo menor en su atención posterior y mejora de la calidad de la atención y la calidad de vida de las gestantes y sus hijos e hijas (Alfaro y Campos, 2014).

De igual forma, ambos grupos tuvieron ingesta de medicamentos como suplementos vitamínicos, acetaminofén, tratamiento para detener el vómito; pero en mayor proporción el grupo de un CI mayor a 70. Por el contrario, no se encontró la presencia de un consumo de cigarrillo
- marihuana en las madres durante el embarazo en ninguno de los grupos. El consumo de alcohol en algunas ocasiones fue mayor en un $61,1 \%$ en el grupo de un CI mayor a 70, a comparación con el $38,9 \%$ en el grupo de un CI menor a 70 , lo que no representa a nivel prenatal factor de riesgo. En cuanto a la exposición prenatal al alcohol, que fue uno de los elementos de estudio presentados en esta investigación, se encontró una significancia de 0,395, lo cual explica que no se encontrara una asociación directa con el CI, en comparación con el estudio realizado en Estados Unidos por Vaurio, Riley y Mattson (2011), quienes sí encontraron asociación. La literatura, por su parte, ha demostrado que las madres que consumen alcohol durante el embarazo generan una exposición a los hijos que puede traer problemas a nivel cerebral y conductual (Papalia, Wendkos y Duskin. 2009).

En cuanto al consumo de cocaína, esta investigación no presentó asociación directa a nivel prenatal con el CI. Esto coincide con los resultados obtenidos por Singer, et al. (2005), quienes encontraron que no existe una asociación directa. Además, los resultados de esta investigación coindicen con otras investigaciones en las que la exposición a la cocaína no se asocia con el funcionamiento intelectual en los niños y niñas en años escolares (Frank et al., 2004; Wasserman et al., 1998).

Por otra parte, se encontró que una de las enfermedades presentes durante el embarazo fue la infección urinaria, presentándose en un $72,7 \%$ para el grupo de un CI menor a 70 y un 27,3\% para el grupo de un CI mayor a 70 . Esto coincide con la investigación realizada por Arrieta, Ballestas, García, Jiménez y Medina (2013), quienes encontraron índices altos a partir de los cambios fisiológicos que se presentan en este estado, haciendo a las mujeres más vulnerable a padecer 
este tipo de infección; sin embargo, esta investigación muestra que a pesar de la alta frecuencia de esta enfermedad, no presenta ninguna asociación con el CI.

Frente a las variables intraparto, se encontró un mayor acercamiento al uso de anestesia durante el proceso de parto; sin embargo, es común que en los procesos de embarazo se utilice la anestesia, especialmente para los partos realizados por cesárea, ya que en Colombia, entre un $25 \%$ y $30 \%$ de los partos se realizan de esta manera (Páez y Navarro, 2012); y en algunos casos se recurre a este procedimiento para realizar una ligadura de trompas para no tener embarazos (Río Fortuna, 2007).

La Organización Mundial de la Salud (OMS) menciona que las tres principales causas de muerte de niñas y niños recién nacidos en el mundo son las infecciones, la prematuridad y la asfixia perinatal o complicaciones del parto, que constituyen el $23 \%$ de la mortalidad neonatal global (Kurinczuk, White-Koning, y Badawi, 2010). En este estudio, no se evidenció diferencia alguna. Este resultado contrasta con el estudio realizado por Ibarraran, González, González y Hernández (2001), quienes agregaron que la hipoxia perinatal es una de las causas de un bajo CI en niños y niñas en la etapa preescolar que obtuvieron un puntaje de CI menor de ochenta, y que presentaron mayores dificultades en la expresión verbal.

Ahora bien, el bajo peso al nacer (BPN) es un problema de salud pública mundial, que constituye el principal factor de mortalidad perinatal (Parra, Rodríguez y Chinome, 2015). Para Portellano (2007) el BPN se presenta cuando el neonato evidencia un peso entre 1500 y 2500 gramos. En esta investigación no se encontró evidencia de esto y contrasta con varios estudios que conducen a lo mismo, como el estudio que encontró que los niños y niñas en etapas preescolares que tuvieron un coeficiente intelectual menor de 70 presentaron un peso entre $750 \mathrm{~g}$ y $1499 \mathrm{~g}$ (Velázquez, Masud y Ávila, 2004). O el estudio que dice que el peso al nacer puede influir en el desarrollo del neonato a lo largo de su vida (Velázquez et al., 2004) y otros que mencionan que está relacionado con la presencia de coeficiente intelectual limítrofe, manifestado en el largo plazo (Atuesta, Vásquez y Urrego, 2008).

\section{Conclusiones}

La presente investigación buscó determinar los factores prenatales y perinatales asociados al coeficiente intelectual en niños y niñas que asisten a hogares comunitarios en el barrio Uribe UribeSincelejo, intentando establecer un nivel de influencia en el CI. Se concluye que no existe asociación entre las variables que contemplan los factores pre y perinatales en el desarrollo del CI en niños del barrio Uribe, Uribe, periferia de Sincelejo.

Seguidamente, es de resaltar que aunque, si bien la literatura en muchas ocasiones nos dota de una asociación relevante de estos factores en desarrollo intelectual, se concluye que estadísticamente no se evidencia relación alguna al menos en esta población; siendo que a pesar de ser un barrio con algunas demandas económicas, sociales y educativas, no constituyen en sí una vulnerabilidad de esta población hacia en bajo CI.

En concordancia con lo anterior, se puede afirmar que el desarrollo del $\mathrm{CI}$, puede estar ligado a otras variables, por lo que se recomienda seguir ahondando en futuras investigaciones en otros factores como bien lo puede ser el contexto familiar, escolaridad de los cuidadores, el grado de estimulación en los primeros meses y años de vida, donde quizás se encuentre algún tipo de asociación. 
Rada-Luna et al. Factores prenatales y perinatales asociados al coeficiente intelectual en niños (a) de tres a seis años

\section{Referencias bibliográficas}

Alfaro, N. y Campos, G. (2014). Análisis del control prenatal que se brinda a las gestantes de la provincia de Heredia que tienen su parto en el hospital San Vicente de Paúl. Revista enfermería actual en Costa Rica, 26, 1-19.

Arrate, M.; Linares, M.; Molina, V.; Sánchez, N. y Arias, M. (2013). Efectos secundarios de los anticonceptivos hormonales en usuarias del método asistentes a las consultas de planificación familiar. Medisan, 17(3), 415-425.

Arrieta, N.; Ballestas, M.; García, G.; Jiménez, O. y Medina, J. (2013). Prevalencia de infección urinaria en pacientes gestantes atendidas en el programa de control prenatal en el Hospital Materno Infantil de Soledad durante el año 2012. Revista Médica Evidencias, 3(1), 37-43.

Atuesta, J.; Vásquez, R. y Urrego, Z. (2008). Aspectos psicopatológicos del coeficiente intelectual limítrofe: un estudio en el Hospital de la Misericordia, 2000-2005. Revista Colombiana de Psiquiatría, 37(2), 182- 194.

Bernal, D. y Cardona, D. (2014). Caracterización de la mortalidad perinatal en Manizales, Colombia, 2009- 2012. Hacia la promoción de la salud, 19(2): 66-80.

Bolzán, A. y Guimarey, M. (2001). Relación entre el índice de masa corporal durante la gestación en embarazadas adolescentes y adultas, indicadores antropométricos de crecimiento fetal y retardo de crecimiento intrauterino. La Costa, Argentina, 1999. Archivos Latinoamericanos de Nutrición, 51(2), 145-150.
Cadavid, M.; Zapata, M.; Aguirre, D. y Álvarez, M. (2011). Coeficiente intelectual de niños escolarizados en instituciones públicas de las zonas nororiental y noroccidental de Medellín según el nivel de seguridad alimentaria del hogar y condiciones socioeconómicas. Revista Chilena de Nutrición, 38(4), 392-403.

Catalano, P. (2007). Management of Obesity in Pregnancy. Obstetrics $y$ Gynecology, 109(2), 419-433.

Departamento Administrativo Nacional de Estadística. (2012). Pobreza monetaria y multidimensional 2012. Recuperado de: https://www. dane.gov.co/files/investigaciones/ condiciones_vida/pobreza/cp_ pobreza_departamentos_2012.pdf

Derauf, C., LaGasse, L., Smith, L., Grant, P., Shah, R., Arria, A., et al. (2007). Demographic and psychosocial characteristics of mothers using methamphetamine during pregnancy: preliminary results of the infant development, environment, and lifestyle study (IDEAL). The American Journal of Drug and Alcohol Abuse, 33(2), 281-289. doi: $10.1080 / 00952990601175029$

Dow-Edwards, D., Mayes, L., Spear, L. y Hurd, Y. (1999). Cocaine and development: clinical, behavioral, and neurobiological perspectives - a symposium report. Neurotoxicology and Teratology, 21(5), 481-490.

Evans, G. y Kantrowitz, E. (2002). Socioeconomic status and health: the potential role of environmental risk exposure. Annual Review of Public Health, 23, 303-331. doi:10.1146/annurev. publhealth.23.112001.112349. 
Feng, M., Yan, S. y Yan, Q. (2005). Effects of prenatal alcohol exposure on brain-derived neurotrophic factor and its receptor tyrosine kinase $B$ in offspring. Brain Research, 1042(2), 125-132. doi: 10.1016/j. brainres.2005.02.017

Ferrer, R. y Torres, L. (2014). Análisis comparativo entre el coeficiente intelectual y el desempeño en una tarea de solución de problemas en estudiantes de primaria con éxito y fracaso escolar, [Tesis de pregrado]. Recuperado de: https:// repository.javeriana.edu.co/ bitstream/handle/10554/19104/ FerrerFerrerRaquelCecilia2014. pdf? sequence $=1$ yisAllowed $=y$

Flores-Compadre, J.; Cruz, F.; Orozco, G. y Vélez, A. (2013). Hipoxia perinatal y su impacto en el neurodesarrollo. Revista chilena de neuropsicología, 8(1), 26-31.

Frank, D.; Rose-Jacobs, R.; Beeghly, M.; Wilbur, M.; Bellinger, D. y Cabral, H. (2004). Level of prenatal cocaine exposure and 48-month IQ: importance of preschool enrichment. Neurotoxicology and Teratology, $27(1), \quad 15-28$. doi: $10.1016 / j$. ntt.2004.09.003.

Gaspar, F., Harley, K., Kogut, K., Chevrier, J., Mora, A., Shödin, A. et al. (2015). Prenatal DDT and DDE exposure and child IQ in the CHAMACOS cohort. Environment International, 85, 206-212. doi: 10.1016/j. envint.2015.09.004

Glover, V. (2011). The effects of prenatal stress on child behavioural and cognitive outcomes start at the beginning. Recuperado de: http:// www.child-encyclopedia.com/sites/ default/files/textes-experts/en/872/ the-effects-of-prenatal-stress-on- child-behavioural-and-cognitiveoutcomes-start-at-the-beginning.pdf

González de Dios, J.; Moya, M. y Vioque, J. (2001). Factores de riesgo predictivos de secuelas neurológicas en recién nacidos a término con asfixia perinatal. Revista de Neurología, 32(3), 210-216.

Goya, M.; Flores, C.; Astudillo, R.; Viso, C. y Cabero, L. (2008). Obesidad y su impacto perinatal. Folia Clínica en Obstetricia y Ginecología, 72, 6-31.

Gunderson, E. y Abrams, B. (2000). Epidemiology of gestational weight gain and body weight changes after pregnancy. Epidemiologic Reviews, 22(2), 261-274.

Harmony, T. (1996). Factores que inciden en el desarrollo del sistema nervioso de niño; Cap. 8. En Aproximaciones de las neurociencias a la conducta. De Corsi-Cabrera, M. México, Universidad de Guadalajara UNAM.

Herring, S. y Oken, E. (2010). Ganancia de peso durante el embarazo: su importancia para el estado de salud materno-infantil. Annales Nestlé, 68, 17-28. doi: 10.1159/000320346.

Huang, Z. J., Di Cristo, G. y Ango, F. (2007). Development of GABA innervation in the cerebral and cerebellar cortices. Nature Reviews Neuroscience, (8), 673-686. doi: $10.1038 / \mathrm{nrn} 2188$

Ibarraran, M.; González, Y.; González, C. y Hernández, J. (2001). Influencia de la hipoxia perinatal sobre el desarrollo en la etapa preescolar. Revista mexicana de neurociencia, 2(5), 281-287.

Jacobson, L. y Jacobson, W. (2002). Effects of prenatal alcohol exposure on child development. Alcohol Research y Health, 26(4), 282-286. 
Rada-Luna et al. Factores prenatales y perinatales asociados al coeficiente intelectual en niños (a) de tres a seis años

James, A. y Cherian, S. (2010). Pathophysiology of perinatal hypoxiaischaema. Paediatrics and Child Health, 20(8), 351-355.

Kurinczuk, J.; White-Koning, M. y Badawi, N. (2010). Epidemiology of neonatal encephalopathy and hypoxic-ischaemic encephalopathy. Early Human Development, 86, 329-338. doi: 10.1016/j. earlhumdev.2010.05.010.

Lambe, M., Hultman, C., Torrång, A., MacCabe, J. y Cnattingius, S. (2006). Maternal smoking during pregnancy and school performance at age 15. Epidemiology, 17(5), 524-530. doi:10.1097/01. ede.0000231561.49208.be

Landon, M., Hauth, J., Leveno, K., Spong, C., Leindecker, S., Varner, M., et al. (2004). Maternal and perinatal outcomes associated with a trial of labor after prior cesarean delivery. $\mathrm{N}$ Engl J Med, 351, 2581-2589. DOI: 10.1056/NEJMoa040405.

Langley, K., Rice, F., Van den Bree, M. B. y Thapar, A. (2005). Maternal smoking during pregnancy as an environmental risk factor for attention deficit hyperactivity disorder behaviour. A review. Minerva Pediatrica, 57(6), 359-372.

Lanphear, B., Hornung, R., Khoury, J., Yolton, K., Baghurst, P., Bellinger, D. et al. (2005). Low-level environmental lead exposure and children's intellectual function: an international pooled analysis. Environmental Health Perspectives, 113(7), 894-899.

Lara, D.; Utria, O. y Ávila, J. (2012). Factores de riesgo pre, peri y postnatales asociados al género en niños y niñas con autismo.
International Journal of Psychological Research, 5(2), 77-90.

Martin, R. y Dombrowski, S. (2008). Prenatal Exposures: Psychological and Educational Consequences for Children. New York, Estados Unidos: Springer.

Mattson, S., Crocker, N. y Nguyen, T. (2011). Fetal alcohol spectrum disorders: neuropsychological and behavioralfeatures. Neuropsychology Review, 21(2), 81-101.

Miller, M. (2003). Expression of transforming growth factor-beta in developing rat cerebral cortex: Effects of prenatal exposure to ethanol. The Journal of Comparative Neurology, 460, 410-424.

Ministerio de Salud. (2013). Guía de Práctica Clínica (GPC) para la prevención, detección temprana y tratamiento de las complicaciones del embarazo, parto o puerperio. Recuperado de: http://gpc.minsalud. gov.co/gpc_sites/Repositorio/ Conv_500/GPC_embarazo/GPC_ embarazo_completa.aspx

(2016). Análisis de Situación en Salud (ASIS) Colombia, 2016. Recuperado de https://www. minsalud.gov.co/sites/rid/Lists/ BibliotecaDigital/RIDE/VS/ED/PSP/ asis-colombia-2016.pdf

Minjarez, M.; Rincón, I.; Morales, Y.; Espinosa, M.; Zárate, A. y Hernández, M. (2014). Ganancia de peso gestacional como factor de riesgo para desarrollarcomplicaciones obstétricas. Perinatología y reproducción humana, 28(3), 159-166.

Moreno, J.; Yáñez, G.; Prieto, B.; Rodríguez, Y. y García, A. (2017). Una revisión de la literatura acerca de las características neuropsicológicas de 
niños con craneosinostosis simple en diferentes edades. Revista chilena de neuro-psiquiatría, 55(1), 52-63.

Oken, E.; Taveras, E.; Kleinman, K.; RichEdwards, J. y Gilman, M. (2007). Gestational weight gain and child adiposity at age 3 years. American journal of obstetrics y gynecology, 196(4), 322.e1-322.e8.

Olson, C. (2008). Achieving a healthy weight gain during pregnancy. Annual Review of Nutrition, 28, 411-423. doi: 10.1146/annurev. nutr.28.061807.155322.

Strawderman, M. y Dennison, B. (2009). Maternal weight gain during pregnancy and child weight at age 3 years. Maternal and child health journal, 13(6), 839-846.

Páez, J. y Navarro, R. (2012). Anestesia regional versus general para parto por cesárea. Revista Colombiana de Anestesiología, 40(3), 203-206.

Parra, J.; Rodríguez, L. y Chinome, J. (2015). Relación entre peso al nacer y madurez neuropsicológica en preescolares de Tunja (Colombia). Pensamiento Psicológico, 13(2), 65-77.

Portellano, J. (2007). Neuropsicología infantil. Madrid, España: Editorial Síntesis.

Rijlaarsdam, J.; Cecil, C.; Walton, E.; Mesirow, M.; Relton, C.; Gaunt, T. et al. (2017). Prenatal unhealthy diet, insulin-like growth factor 2 gene (IGF2) methylation, and attention deficit hyperactivity disorder symptoms in youth with early-onset conduct problems. Journal of child psychology and psychiatry, 58(1), 19-27.

Río Fortuna, C. (2007). El acceso a la ligadura de trompas de Falopio en la ciudad de Buenos Aires: la maternidad responsable y saludable frente al derecho a decidir sobre el propio cuerpo. Cuadernos de Antropología Social, 25, 169-188.

Salazar, L. (2010). Autonomía personal y salud infantil. Málaga, España: Innovación y Cualificación.

San Román, M. (2013). Aumento del peso durante el embarazo: modificaciones fisiológicas relacionadas con la ganancia de peso y necesidades nutricionales, [tesis de pregrado]. Recuperado de: https://repositorio.unican.es/xmlui/ bitstream/handle/10902/3948/ $\mathrm{S}$ a $\mathrm{n} R \circ \mathrm{m}$ a $\mathrm{n} \mathrm{D}$ i e g $\mathrm{g} \mathrm{MA}$. pdf? sequence $=1 \mathrm{yis}$ Allowed $=\mathrm{y}$

Sánchez, H.; Pérez, G.; Pérez, P. y Vásquez, F. (2005). Impacto del control prenatal en la morbilidad y mortalidad neonatal. Revista Instituto Mexicano del Seguro Social, 43(5), 377-380.

Schnaas, L., Rothenberg, S., Perroni, E., Martínez, S., Hernández, C. y Hernández, R. (2000). Temporal pattern in the effect of postnatal blood lead level on intellectual development of young children. Neurotoxicology and Teratology, 22(6), 805-810.

Secretaria de Planeación de Sincelejo. (2016). Plan de desarrollo 2016-2019 "Ciudad con visión". Recuperado de:http://www. alcaldiadesincelejo. gov.co/Ci u d a d a nos / ProyectosNormatividad/PIan $\% 20$ de $\% 20$ Desarrollo\%20Municipal\%20 2016\%20-\%202019.pdf

Shen, X., Yan, C., Guo, D., Wu, S., Li, R., Huang, H. et al. (1998). LowLevel prenatal lead exposure and neurobehavioral development of children in the first year of life: 
Rada-Luna et al. Factores prenatales y perinatales asociados al coeficiente intelectual en niños (a) de tres a seis años

A prospective study in Shanghai. Environmental Research, 79(1), 1-8.

Singer, L.; Eisengart, L.; Minnes, S.; Noland, J.; Jey, A.; Lane, C. et al. (2005). Prenatal cocaine exposure and infant cognition. Infant Behavior y Development, 28(4), 431-444.

Thompson, B.; Levitt, P. y Stanwood, G. (2009). Prenatal exposure to drugs: effects on brain development and implications for policy and education. Nature Reviews Neuroscience, 10(4), 303-312.

Torres, P. y Granados, D. (2010) Factores de riesgo perinatal, signos neurológicos blandos y lenguaje en edad preescolar. Enfermeria Neurológica, 12(3), 128-133.

Valenzuela, C. y Trujillo, S. (1984). Coeficiente intelectual y edad de menarquía en niñas escolares del área norte de Santiago. Revista chilena de pediatría, 55(3), 184-187.

Vaurio, L.; Riley, E. y Mattson, S. (2011). Neuropsychological comparison of children with heavy prenatal alcohol exposure and an IQ-Matched comparison group. Journal of the International Neuropsychological Society, 17(3), 463-473.

Velázquez, N.; Masud, J. y Ávila, R. (2004). Recién nacidos con bajo peso; causas, problemas y perspectivas a futuro. Boletín médico del Hospital Infantil de México, 61(1), 73-86.

Villatoro, R. (2016). Correlación entre coeficiente intelectual $y$ coeficiente emocional en las niñas de segundo grado primaria, del Centro Escolar Entrevalles, municipio Fraijanes, [Tesis de pregrado]. Recuperado de:http:// biblioteca.galileo.edu/tesario/ bitstream/123456789/365/1/
Roxana\%20Arely\%20Villatoro\%20 Higueros.pdf

Vishnevetsky, J., Tang, D., Chang, H., Roen, E., Wang, Y., Rauh, V., et al. (2015). Combined effects of prenatal polycyclic aromatic hydrocarbons and material hardship on child IQ. Neurotoxicology and Teratology 49, 74-80.

Viswanathan M.; Siega-Riz, A.; Moos, M.; Deierlein, A.; Mumford, S.; Knaack, J. et al. (2008). Outcomes of Maternal Weight Gain. Evidence Report/ Technology Assessment, 168, 1-223.

Wasserman, G.; Kline, J.; Bateman, D.; Chiriboga, C.; Lumey, L.; Friedlander, $\mathrm{H}$. et al. (1998). Prenatal cocaine exposure and school-age intelligence. Drug and Alcohol Dependence, 50(3), 203-210.

Wasserman, G., Liu, X., Popovac, D., Factor-Litvak, P., Kline, J., Waternaux, C. et al. (2000). The Yugoslavia prospective lead study: contributions of prenatal and posnatal lead exposure to early intelligence. Neurotoxicology and Teratology, 22(6), 811-818.

Wechsler, D. (1973). La medida de la inteligencia del adulto. Buenos Aires, Argentina: Ediciones Huascar.

Zapata, M.; Álvarez, M.; Aguirre, D. y Cadavid, M. (2012). Coeficiente intelectual y factores asociados en niños escolarizados en la ciudad de Medellín, Colombia. Revista de Salud Pública, 14(4), 543-557. Recuperado de: https://revistas.unal.edu.co/ index.php/revsaludpublica/article/ view/20522/40320: :pdf 